\title{
The Impact of Repeated Lidocaine Injections, Exercise, and Mindfulness on Fibromyalgia Symptoms in a Clinical Population: A Pilot Study
}

\section{Grace Hansen}

College of Public Health and Health Professions

Faculty Mentor: Roland Staud, Department of Medicine

\begin{abstract}
Fibromyalgia (FM) patients usually are resistant to conventional single mode therapy. Therefore, the efficacy of combined pharmacological and nonpharmacological treatments for the management of FM symptoms in a clinical population was assessed. Participants were injected with $4 \times 5 \mathrm{~mL} 1 \%$ lidocaine $\mathrm{HCl}$ solution and given education materials and support on increasing their daily steps walked and to engage in mindfulness techniques to reduce their overall pain and accompanying symptoms of their condition. Participants self-reported pain intensity, unpleasantness, anxiety, fatigue, and depression three days prior to and after each injection. Overall ratings of pain intensity and unpleasantness showed statistically significant reductions of 34.7 and 47.2 percent respectively with the combined use of treatments. Additionally, fatigue levels had a significant decrease but depression and anxiety ratings were not significantly modified by this treatment modality. Patients who had a greater adherence to their protocol had an improved pain relief when compared to others. These results suggest that the use of a combination treatment method for FM in a clinical setting and when used in combination is effective. This pilot study can be used as a model for future randomized controlled studies on comprehensive treatments for FM.
\end{abstract}

Keywords: fibromyalgia, multidisciplinary, lidocaine, exercise, mindfulness

\section{Introduction}

Fibromyalgia (FM) is a chronic illness that is characterized by widespread musculoskeletal pain and by at least 11 out of 18 tender points, located in designated bodily areas as required by The American College of Rheumatology 1990 FM Criteria (Wolfe et al.,1990). Additional symptoms include chronic fatigue, tenderness, stiffness, memory deficits, sleep disturbances, bowel and bladder dysfunctions, anxiety, and depression. FM is much more common in women, with around 80 to 90 percent of diagnoses being detected in women (Arnold et al., 2011). FM can cause a significant impairment in one's daily function both physically and psychologically and can often impair one's overall quality of life. 
The mechanisms behind FM are currently not well understood but include central nervous system abnormalities. Standard treatment as designated by EULAR includes a graded and individualized approach, beginning with patient education and non-pharmacological therapies, followed by the use of pharmacological therapies if necessary (Mcfarlane et al., 2016). The focus of this treatment recommendation is directly on improving health-related quality of life (HRQoL) for individuals with FM. Similarly, a systematic review of treatments regarding FM using the American College of Rheumatology criteria has suggested that the combination of pharmacological and non-pharmacological treatments may be most beneficial for patients that is tailored to each individual's pain (Carville et al, 2008).

In terms of a pharmacological treatment, the use of lidocaine injections when compared to saline injections in patients with FM has proven to effectively reduce their overall fatigue and increase their mechanical pain thresholds (Staud et al., 2017). Non-pharmacological treatment methods such as physical exercise regiments, psychosocial, and dietary interventions in a holistic manner have all proven to be important considerations for treatment (Derman et al., 2011). An important aspect of this approach is the patient-specific adjustments to these treatments. An overall improved HRQoL for those with FM was found when combining a multidisciplinary approach in combination with analgesics or antidepressants (Jacobs et al., 2019). With this research in mind, interventions that are both holistic, supportive, and individually tailored to the patient may be the most effective treatment for FM patients. This pilot study is the first of its kind to examine the effectiveness of a combination of repeated lidocaine injections, physical exercise, mindfulness, and counseling with follow-ups on a clinical population of FM patients, as well as evaluate patient adherence to a personalized treatment plan.

Most studies have used a single approach of both pharmacological and nonpharmacological treatments for FM patients. Therefore, this study aims to, examine the effects of a combination of repeated trigger point injections with lidocaine, exercise, and mindfulness with personalized education and support for patients with FM, assess if there is a relationship between number of steps and completing daily mindfulness techniques with a reduction in overall pain intensity and pain unpleasantness, and monitor adherence to an individualized treatment plan for FM patients. It was hypothesized that the pain of patients would improve when following an individualized, multidisciplinary plan of treatment that involves both pharmacological and nonpharmacological 
treatments. With a reduction in pain, it was also hypothesized that a reduction in fatigue, depression, and anxiety would also occur.

\section{Methods}

The data for this study was collected during clinical visits of FM patients at The Department of Medicine in the Division of Rheumatology at Medical Plaza at the University of Florida. The use of these data for study purposes was approved by the University of Florida Institutional Review Board under consent \#201600816. The protocol conformed to the ethical guidelines of the 1975 Declaration of Helsinki. Inclusion criteria included 1) adults over the age of $18 ; 2$ ) the ability to provide informed consent; 3 ) patients were diagnosed with FM using the American College of Rheumatology 1990 and 2011 Criteria; 4) a patient score greater than 12 on the Fibromyalgia Distress Scale; and 5) willingness to adhere to the treatment protocol. The study population included 6 patients that were all female, with the mean age being 53.6 years and enrollment information shown in the diagram below (Eldridge et al., 2010).

\section{$=\mathrm{CONSORT}$}

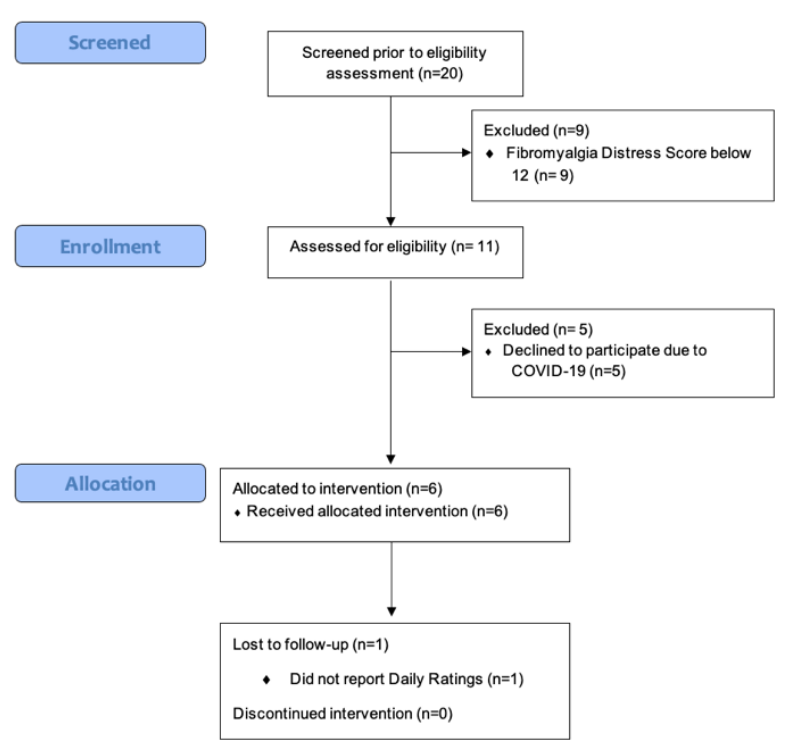

Participants completed a daily pain survey, the Fibromyalgia Impact Questionnaire (FIQ) and Daily Pain Ratings including pain intensity, pain unpleasantness, fatigue, depression, anxiety, steps walked, and if they engaged in mindfulness activities three days before and three 
days after the injection. Patients were educated on the difference between pain intensity and pain intensity using a metaphor for music created by Price et al., which compares pain intensity as the loudness of the sound and pain intensity would be described as the quality of the music (1983).

Prior to the injections, participants completed the Body Pain Diagram and tender points examination, which were were performed by trained study staff members. Patients additionally completed the Pennebaker Inventory of Limbic Languidness (PILL), Patient Global Impression of Disease Activity, and Mechanical Thresholds testing using a Visual Analog Scale (0-10) which are validated and widely accepted scales, examinations, and questionnaires used in chronic pain and specificially in fibromyalgia research which were collected electronically through a secure data collection system (Bennett et al., 2009; Croft et al., 1944; McDermid et al., 1996; Price et al., 1983). The Visual Analog Scale designates a 0 as being "no pain/fatigue at all" on the left and on the right a 100 as being "the most intense pain/fatigue imaginable". Mechanical Thresholds testing utilized an algometer to apply pressure to 1) the trapezius muscles and 2) the upper medial quadrant of the gluteal muscles. The pressure was increased at $0.5 \mathrm{~kg} / \mathrm{s}$ until the participant reported the pressure turning to pain. The average of two tests were used as a baseline for standardized supra-threshold pressure pain testing (avg. baseline pressure x 1.5). During this test the patients immediately reported their pain intensity on the VAS scale followed by rating pain 15 seconds after pressure application. This test was completed immediately before and then again after the lidocaine injections and was used to compare pain intensity relief and fatigue changes due to the injection.

Patients were then injected by the study physician with $5 \mathrm{~mL}$ of $1 \%$ (50 mg) of lidocaine in both centers of the trapezius and lateral to the dimples of Venus (fossae lumbales laterales) into the gluteus maximus muscle bilaterally using a $27 \mathrm{~g}$ hypodermic needle that reached at least one inch into muscle tissue for a total of four injections, as shown in Figure 1 below. The injections were completed over a two minute period while the participant's vital signs and electrocardiogram (ECG) were monitored. 


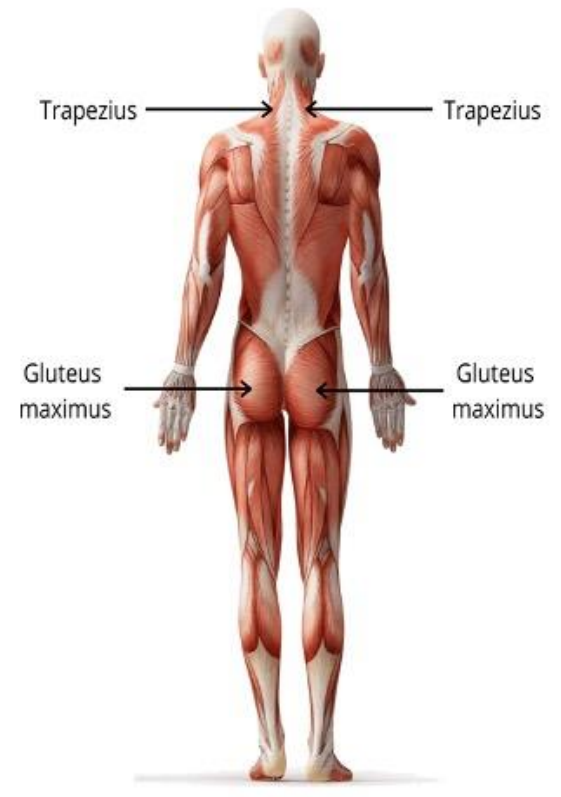

Figure 1. Injection Location Sites

These sites for injections were chosen due to being common areas of muscle pain among FM patients, their considerable size, and accessible position (Staud et al., 2017). Following the injections, Mechanical Pain Thresholds testing was repeated as well as the Body Pain Diagram after 5 minutes to assess the immediate effects of the lidocaine injections. Patients were monitored both before and after injections and provided education by trained study staff to improve the outcome of their injection by engaging in exercise and mindfulness. Participants were provided with resources to gradually increase their daily steps to improve pain levels and other related symptoms and reported steps walked through step-counting applications on mobile devices or smart watches. Information and support was also provided on engaging in daily mindfulness for at least 20 minutes. Informal mindfulness practices were introduced to all participants, including an attention-focusing technique which involves a body scan that directs attention throughout the body in a relaxed state as well as sitting medication that systematically directs attention to breath and immediate sensory and cognitive experiences. Weekly calls were completed with participants to provide additional support and to discuss steps walked and daily completion of mindfulness. Lidocaine injections were repeated by the study physician following at least a period of two weeks in individuals with minor pain relief in order to improve patients pain levels and overall HRQoL. 
Finally, descriptive, multivariate statistical analysis was used to quantify data and examine relationships between pain, fatigue, depression, anxiety levels, steps walked, and engagement with mindfulness techniques.

\section{Results}

When averages of pain ratings three days before the injection were compared to three days after the injection, there was a 34.7 percent decrease in pain intensity, and a 41.2 decrease in overall pain unpleasantness (Figure 2). While fatigue levels decreased by 40.3 percent, anxiety and depression improved by 14.3 and 13.6 percent which are not statistically significant changes. It was found that the combined effects of pharmacological and nonpharmacological treatments with personalized education and support for patients with FM was an effective treatment model for this pilot study. This study also assessed patient's adherence to an individualized treatment plan and found that those that had a greater percentage of pain reduction followed their treatment plan more closely overall and had a greater percentage of increase between steps per day.

In the three days after the injections, participants had a 55.2 percent increase in steps per day when compared to three days before (Figure 4). In terms of mindfulness, those that engaged in these techniques also showed a greater reduction in pain intensity and unpleasantness compared to those who did not engage in daily mindfulness techniques. The combination of lidocaine injections with engaging in exercise and mindfulness techniques had clinically significant impact on patients' overall pain intensity, unpleasantness, and fatigue levels.

In order to assess the impact of HRQoL, the FIQ and PILL were scored at subsequent visits and quantified in order to evaluate the impact of this pilot study on quality of life measures and activities of daily living. The FIQ measures physical functioning, work status, depression, anxiety, morning tiredness, pain, stiffness, fatigue, and overall well-being and assists in quantifying the quality of life changes for those enrolled in this treatment protocol (Bennett et al., 2009). The PILL was also scored and used to assess overall participants ratings of bodily sensations which can relate to level of distress (McDermid et al., 1996). It was found that those who had a greater increase in their HRQoL followed their personalized treatment plan more closely. 


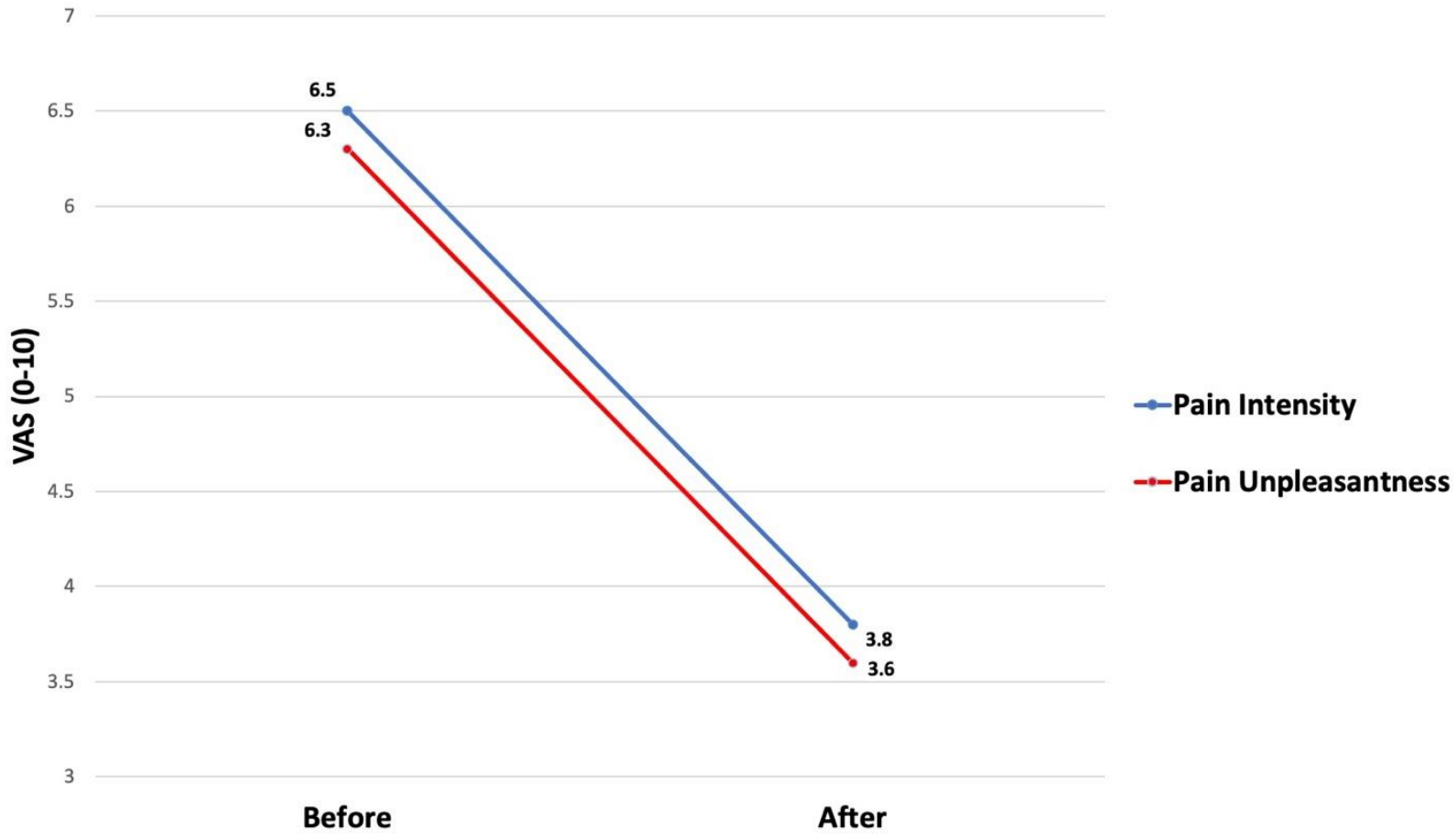

Figure 2. Pain Intensity and Unpleasantness Before and After the Interventions

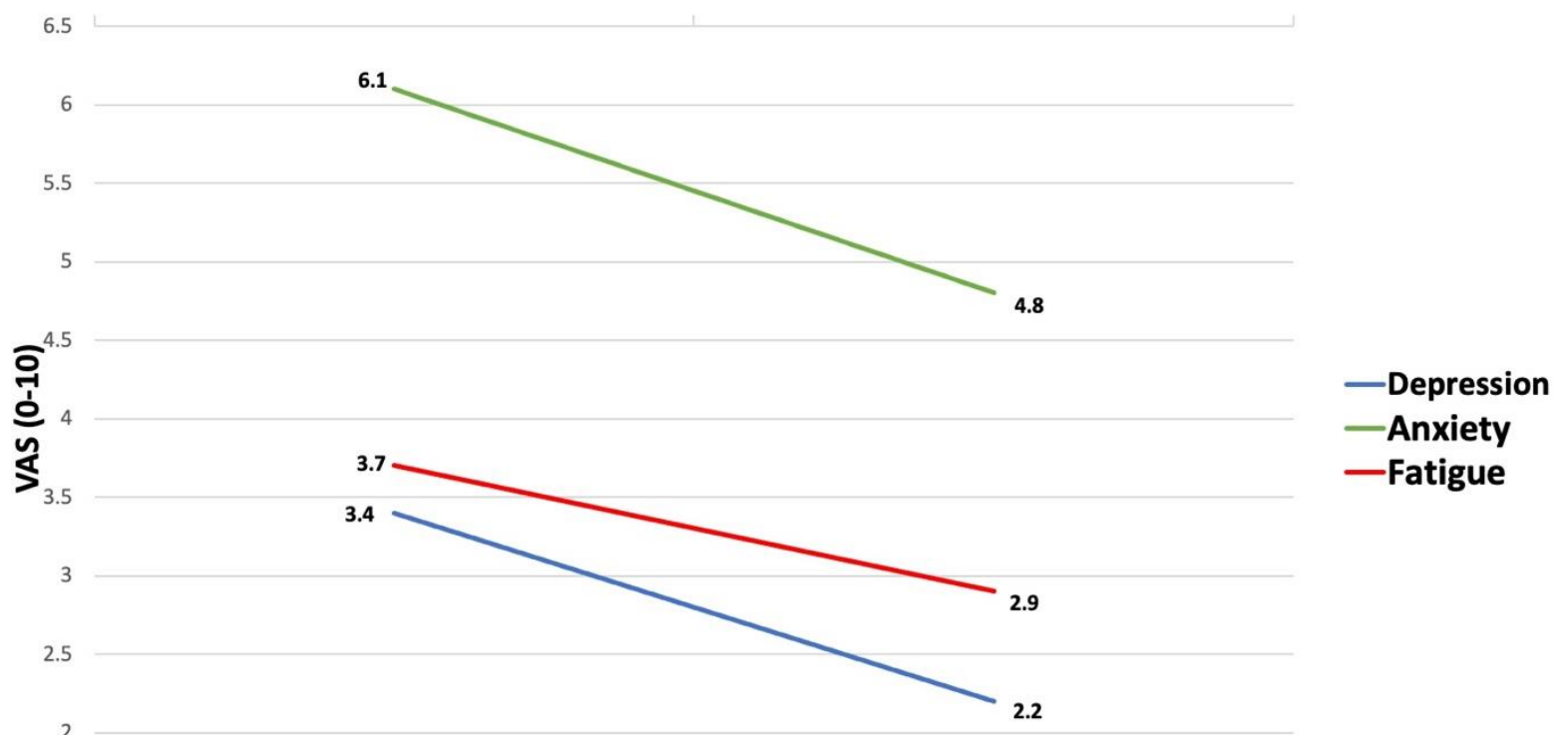

1.5

Before

After

Figure 3. Depression, Anxiety, and Fatigue Before and After the Interventions 


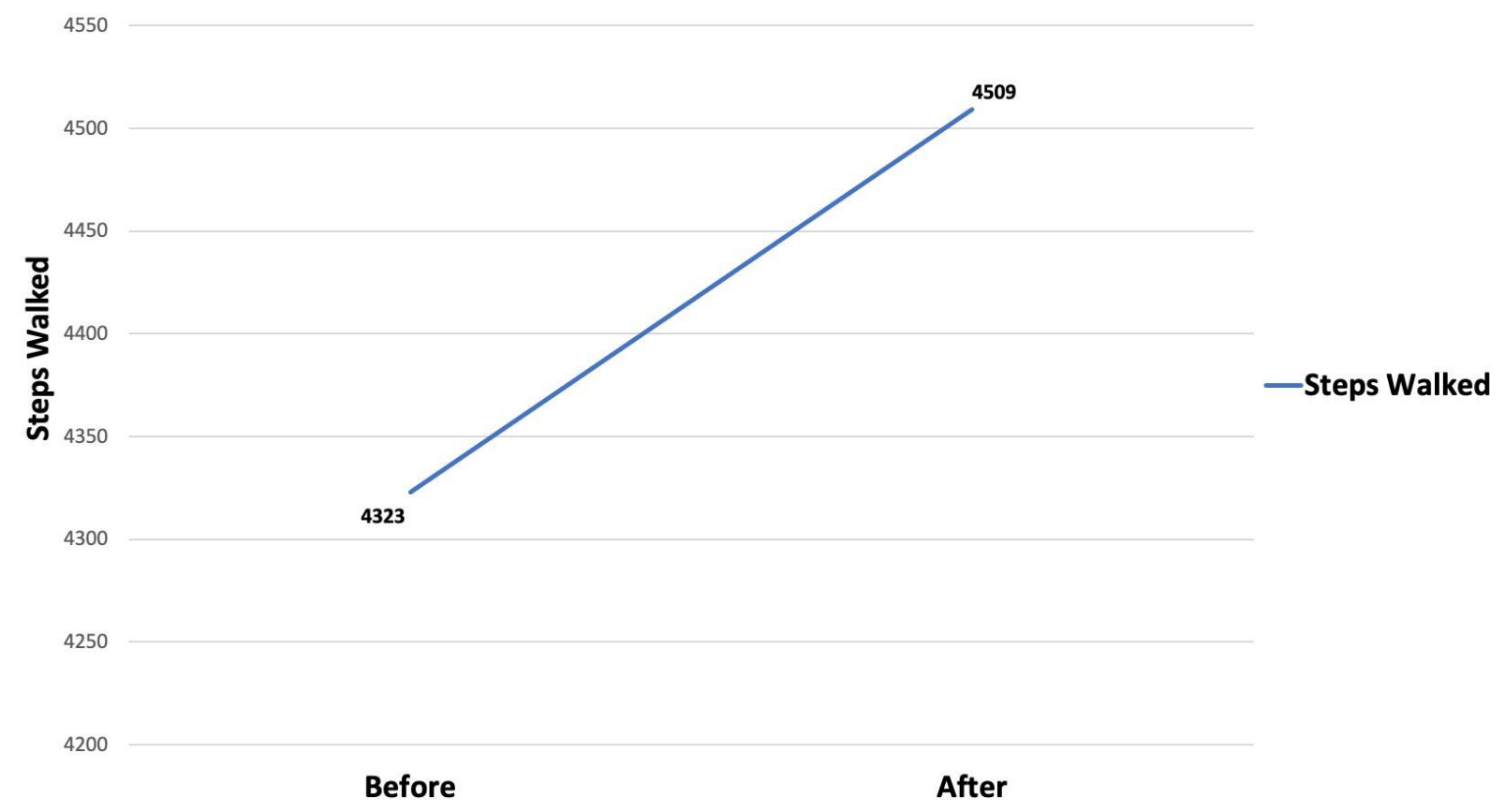

Figure 4. Steps Walked Before and After the Interventions

\section{Discussion}

The hypothesis that the pain of patients would improve when following an individualized, multidisciplinary plan of treatment that involves both pharmacological and nonpharmacological treatments was supported. With a reduction in pain, it was also hypothesized that a reduction in fatigue, depression, and anxiety would also occur. However, clinically meaningful changes occurred in ratings of fatigue levels, while anxiety and depression levels did not differ significantly as shown in Figure 3. The results show that a combined and individualized approach using both pharmacological and nonpharmacological treatments is a promising method for managing pain and fatigue levels of FM patients. It is generally accepted that clinically meaningful improvements on a pain scale of 0 to 10 would be a two-point decrease or a thirtypercent reduction overall (Rowbotham, 2001). Our results would fall into this category of being a clinically meaningful reduction. While this distinction is often used for meta-analyses, the application of this to the small sample size of the study is applicable due to our use of clinical population of participants.

This research was motivated by EULAR recommendation to use a multidisciplinary approach tailored to each individual patient using both pharmacological and nonpharmacological 
treatment and their lack of data from such trials to ensure that this treatment plan is optimal in a clinical setting and when used in combination (Mcfarlane et al., 2016). Due to a lack of research on adequate treatments for those with FM, this study was the first of its kind to holistically examine the effects of lidocaine injections, exercise, and mindfulness interventions, as other trials have only examined one of these modalities (Derman et al., 2011; Staud et al., 2017; Santos et al., 2020).

Limitations of this study must be acknowledged as well, including a small sample size due to the enrollment period being during the COVID-19 pandemic, an entirely female population, and the lack of a control group for comparison. The approach of this pilot study can be expanded upon in the future in a larger sample size to continue examining the effects of a multifaceted pharmaceutical and nonpharmaceutical approach as a treatment plan for those with FM. Future research can be directed towards assessing additional measures such as activity-related pain as well and assessing the long-term implications of using a multidisciplinary, individualized treatment plan.

\section{Acknowledgements}

This study was supported by funding from the University of Florida College of Medicine. The expert assistance of Dr. Roland Staud and Melyssa Godfrey is gratefully acknowledged.

\section{References}

Arnold, L. M., Clauw, D. J., McCarberg, B. H., \& FibroCollaborative (2011). Improving the recognition and diagnosis of fibromyalgia. Mayo Clinic proceedings, 86(5), 457-464.

Bennett, R. M., Friend, R., Jones, K. D., Ward, R., Han, B. K., \& Ross, R. L. (2009). The revised fibromyalgia impact questionnaire (FIQR): validation and psychometric properties. Arthritis research \& therapy, 11(4), 1-14.

Carville, S. F., Arendt-Nielsen, S., Bliddal, H., Blotman, F., Branco, J. C., Buskila, D., ... \& Choy, E. H. (2008). EULAR evidence-based recommendations for the management of fibromyalgia syndrome. Annals of the rheumatic diseases, 67(4), 536-541.

Croft, P., Schollum, J., \& Silman, A. (1994). Population study of tender point counts and pain as evidence of fibromyalgia. Bmj, 309(6956), 696-699.

Derman, E. W., Whitesman, S., Dreyer, M., Patel, D. N., Nossel, C., \& Schwellnus, M. P. (2011). Healthy lifestyle interventions in general practice: Part 16: Lifestyle and fibromyalgia. South African Family Practice, 53(6), 511-515. 
Eldridge SM, Chan CL, Campbell MJ, Bond CM, Hopewell S, Thabane L, et al. CONSORT 2010 statement: extension to randomised pilot and feasibility trials. BMJ. 2016;355.

Geneen, L. J., Moore, R. A., Clarke, C., Martin, D., Colvin, L. A., \& Smith, B. H. (2017). Physical activity and exercise for chronic pain in adults: an overview of Cochrane Reviews. Cochrane Database of Systematic Reviews, (4).

Jacobs, H., Bockaert, M., Bonte, J., D’Haese, M., Degrande, J., Descamps, L., \& Muller, L. (2019). The Impact of a Group-Based Multidisciplinary Rehabilitation Program on the Quality of Life in Patients With Fibromyalgia: Results From the QUALIFIBRO Study. Journal of clinical rheumatology: practical reports on rheumatic \& musculoskeletal diseases.

Macfarlane, G.J., Kronisch, C., Dean, L.E., et al. EULAR revised recommendations for the management of fibromyalgia. Annals of the Rheumatic Diseases 2017;76:318-328.

McDermid, A. J., Rollman, G. B., \& McCain, G. A. (1996). Generalized hypervigilance in fibromyalgia: evidence of perceptual amplification. PAIN@, 66(2-3), 133-144.

Price, D. D., McGrath, P. A., Rafii, A., \& Buckingham, B. (1983). The validation of visual analogue scales as ratio scale measures for chronic and experimental pain. Pain, 17(1), 45-56.

Rowbotham, Michael C. (2001). What is a 'clinically meaningful' reduction in pain? The Journal of the International Association for the Study of Pain. 131-132.

Santos E Campos, M. A., Párraga-Montilla, J. A., Aragón-Vela, J., \& Latorre-Román, P. Á. (2020). Effects of a functional training programme in patients with Fibromyalgia: A 9-years prospective longitudinal cohort study. Scandinavian Journal of Medicine \& Science in Sports.

Staud, R., Kizer, T., \& Robinson, M. E. (2017). Muscle injections with lidocaine improve resting fatigue and pain in patients with chronic fatigue syndrome. Journal of pain research, 10, 1477.

Silverman, S., Dukes, E. M., Johnston, S. S., Brandenburg, N. A., Sadosky, A., \& Huse, D. M. (2009). The economic burden of fibromyalgia: comparative analysis with rheumatoid arthritis. Current medical research and opinion, 25(4), 829-840. https://doi.org/10.1185/03007990902728456

Venuturupalli, R. S., Chu, T., Vicari, M., Kumar, A., Fortune, N., \& Spielberg, B. (2019). Virtual Reality-Based Biofeedback and Guided Meditation in Rheumatology: A Pilot Study. ACR Open Rheumatology, 1(10), 667-675.

Wolfe, F., Smythe, H.A., Yunus, M.B., et al. The American College of Rheumatology 1990 criteria for the classification of fibromyalgia. Report of the Multicenter Criteria Committee. Arthritis Rheum. 1990;33(2):160-172. 